\title{
Recent developments in quasi-elastic scattering around the Coulomb barrier
}

\author{
K. Hagino \\ Department of Physics, Tohoku University, Sendai 980-8578, Japan
}

\begin{abstract}
We discuss two recent topics on heavy-ion quasi-elastic scattering at energies around the Coulomb barrier. The first topic is an application of quasi-elastic scattering at deep-subbarrier energies to extracting the surface diffuseness parameter of the nucleus-nucleus potential. The second topic is a coupled-channels analysis for the quasi-elastic barrier distribution for the ${ }^{70} \mathrm{Zn}+$ ${ }^{208} \mathrm{~Pb}$ reaction. We show that the coupled-channels calculations which include the multi-phonon excitations in the colliding nuclei reproduce reasonably well the experimental excitation function for quasi-elastic scattering at backward angles and the barrier distribution for this reaction.
\end{abstract}

Keywords: Quasi-elastic scattering, barrier distribution, quantum reflection, coupled-channels method, cold fusion

PACS: $25.70 . \mathrm{Bc}, 25.70 . \mathrm{Jj}, 24.10 . \mathrm{Eq}, 27.70 .+\mathrm{w}$

\section{INTRODUCTION}

The internal structure of colliding nuclei strongly influences heavy-ion collisions at energies around the Coulomb barrier. A well known example is a reaction of a deformed nucleus. In this case, the nucleus-nucleus potential depends on the orientation angle of the deformed nucleus with respect to the beam direction. Assuming that the orientation angle does not change during the collision, the cross section can then be obtained by averaging the contribution from all possible angles [1, 2, 3]. In this picture, the relative motion between the colliding nuclei experiences many distributed potential barriers depending on the orientation angle of the target nucleus, instead of a single barrier. To a good approximation, the concept of barrier distribution can be extended also to systems with a non-deformed target [4, 5, 6, 7], where the distribution originates from the coupling between the relative motion and several intrinsic degrees of freedom such as collective inelastic excitations of the colliding nuclei and/or transfer processes.

In Ref. [8], Rowley, Satchler, and Stelson argued that a barrier distribution can be directly extracted from a measured fusion excitation function $\sigma_{\text {fus }}(E)$, by taking the second derivative of the product $E \sigma_{\text {fus }}(E)$ with respect to the center-of-mass energy $E$, that is, $d^{2}\left(E \sigma_{\text {fus }}\right) / d E^{2}$. This has stimulated many high precision measurements of fusion cross section, so that the second derivative is meaningful [4, 9]. The extracted barrier distributions have revealed that the concept indeed holds and the barrier distribution provides a nice tool to investigate the fusion dynamics of the entrance channel. It was also shown recently that the concept of barrier distribution still retains even in massive systems, such as ${ }^{100} \mathrm{Mo}+{ }^{100} \mathrm{Mo}[10]$.

A similar barrier distribution can be extracted also using the quasi-elastic scattering [11, 12]. The quasi-elastic scattering is a sum of elastic, inelastic, transfer, and breakup 
processes, and is a good counterpart of heavy-ion fusion reaction [2]. A major difference is that the quasi-elastic scattering is related to the reflection probability of the Coulomb barrier, while the fusion is related to the transmission. Since the penetration and reflection probabilities are related to each other due to the flux conservation, similar information can be obtained both from fusion and quasi-elastic scattering.

In this contribution, we discuss two recent theoretical activities on heavy-ion quasielastic scattering at sub-barrier energies. We first present our recent systematic analyses on heavy-ion quasi-elastic scattering at deep-subbarrier energies, in aiming at extracting the surface diffuseness parameter of inter-nuclear potential [13, 14]. We then discuss coupled-channels calculations for the ${ }^{70} \mathrm{Zn}+{ }^{208} \mathrm{~Pb}$ reaction, for which the quasi-elastic barrier distribution has recently been obtained experimentally [15].

\section{QUASI-ELASTIC BARRIER DISTRIBUTIONS}

Before we proceed, let us first summarize the theoretical formulas for quasi-elastic barrier distribution. In the eigenchannel representation of the coupled-channels method, the fusion and quasi-elastic cross sections are given as a weighted sum of the cross sections for uncoupled eigenchannels $[2,4,5,6,6]$. That is,

$$
\begin{aligned}
\sigma_{\text {fus }}(E) & =\sum_{\alpha} w_{\alpha} \sigma_{\text {fus }}^{(\alpha)}(E), \\
\sigma_{\mathrm{qel}}(E, \theta) & =\sum_{\alpha} w_{\alpha} \sigma_{\mathrm{el}}^{(\alpha)}(E, \theta),
\end{aligned}
$$

where $\sigma_{\text {fus }}^{(\alpha)}(E)$ and $\sigma_{\mathrm{el}}^{(\alpha)}(E, \theta)$ are the fusion and the elastic cross sections for a potential in the eigenchannel $\alpha$. Notice that the same weight factors $w_{\alpha}$ appear both in Eqs. (1) and (2). This is a generalization of well-known orientation average formula for a system with deformed target,

$$
\sigma(E)=\int_{0}^{1} d\left(\cos \theta_{T}\right) \sigma\left(E ; \theta_{T}\right)
$$

where $\theta_{T}$ is the orientation of the deformed target and represents a continuous variable for $\alpha$ in Eqs. (1) and (2).

The idea of barrier distribution is led by the fact that the classical cross sections for fusion and quasi-elastic scattering for a single potential barrier are given by

$$
\sigma_{\text {fus }}^{c l(0)}(E)=\pi R_{b}^{2}\left(1-\frac{V_{b}}{E}\right) \theta\left(E-V_{b}\right),
$$

and

$$
\sigma_{\mathrm{el}}^{c l(0)}(E, \pi)=\sigma_{R}(E, \pi) \theta\left(V_{b}-E\right),
$$

respectively[12]. Here, $R_{b}$ and $V_{b}$ are the barrier position and the barrier height for the $s$-wave scattering (thus the scattering angle is set to be $\pi$ in Eq. (5)), respectively, and $\sigma_{R}(E, \pi)$ is the Rutherford cross section. These yield [ 8,11$]$,

$$
D_{\text {fus }}(E) \equiv \frac{d^{2}}{d E^{2}}\left[E \sigma_{\text {fus }}(E)\right]=\sum_{\alpha} w_{\alpha} \pi\left[R_{b}^{(\alpha)}\right]^{2} \delta\left(E-V_{b}^{(\alpha)}\right),
$$




$$
D_{\mathrm{qel}}(E) \equiv-\frac{d}{d E}\left(\frac{\sigma_{\mathrm{el}}(E, \pi)}{\sigma_{R}(E, \pi)}\right)=\sum_{\alpha} w_{\alpha} \delta\left(E-V_{b}^{(\alpha)}\right) .
$$

Evidently, these functions provide information on how potential barrier heights are distributed, and are called fusion and quasi-elastic barrier distributions, respectively. In realistic situations, the quantum (tunneling) effect smears the delta function in Eqs. (6) and (7). Moreover, the effect of nuclear potential has to be taken into account in quasielastic cross sections in Eq. (7) [12]. Nevertheless, from the derivation, it is apparent that the fusion and quasi-elastic barrier distributions behave in a similar way. This is demonstrated in Fig. 5 in Ref. [12] for the ${ }^{16} \mathrm{O}+{ }^{154} \mathrm{Sm}$ system.

In actual experiments, it is impossible to put a detector at a scattering angle $\pi$. One can, however, scale a cross section in energy by taking into account the centrifugal correction. Estimating the centrifugal potential at the distance of closest approach for the Rutherford scattering, $r_{c}$, the effective energy may be expressed as [11]

$$
E_{\text {eff }} \sim E-\frac{\lambda_{c}^{2} \hbar^{2}}{2 \mu r_{c}^{2}}=2 E \frac{\sin (\theta / 2)}{1+\sin (\theta / 2)} .
$$

Therefore, one expects that the function $-d / d E\left(\sigma_{\mathrm{el}} / \sigma_{R}\right)$ evaluated at an angle $\theta$ will correspond to the quasi-elastic barrier distribution at the effective energy given by eq. (8).

\section{INTER-NUCLEUS POTENTIAL AND DEEP-SUBBARRIER QUASI-ELASTIC SCATTERING}

Let us now discuss the application of deep-subbarrier quasi-elastic scattering to the problem of surface diffuseness anomaly in heavy-ion potential [16]. For calculations of elastic and inelastic scattering, which are sensitive only to the surface region of the nuclear potential, the diffuseness parameter of around $0.63 \mathrm{fm}$ has been conventionally employed [17, 18]. This value of surface diffuseness parameter has been well accepted, partly because it is consistent with a double folding potential [19]. In contrast, a recent systematic study has shown that experimental data for heavy-ion fusion reactions require a much larger value of the diffuseness parameter, ranging between 0.75 and $1.5 \mathrm{fm}$, as long as the Woods-Saxon parameterization is employed [20].

Since quasi-elastic scattering and fusion are complementary to each other, it is of interest to investigate this problem using quasi-elastic scattering. In doing so, we are particularly interested in the deep sub-barrier region [13, 14]. At these energies, the cross sections of (quasi-)elastic scattering are close to the Rutherford cross sections, with small deviations caused by the effect of nuclear interaction, $V_{N}$. This effect can be taken into account by the semiclassical perturbation theory [12, 21], which leads to

$$
\frac{d \sigma_{\mathrm{el}}(E, \theta)}{d \sigma_{R}(E, \theta)} \sim 1+\frac{V_{N}\left(r_{c}\right)}{k a} \frac{\sqrt{2 a \pi k \eta}}{E}
$$

where $k=\sqrt{2 \mu E} / \hbar, \mu$ being the reduced mass. $\eta$ is the Sommerfeld parameter, and $r_{c}$ is the distance of closest approach. This formula shows that the deviation of the 


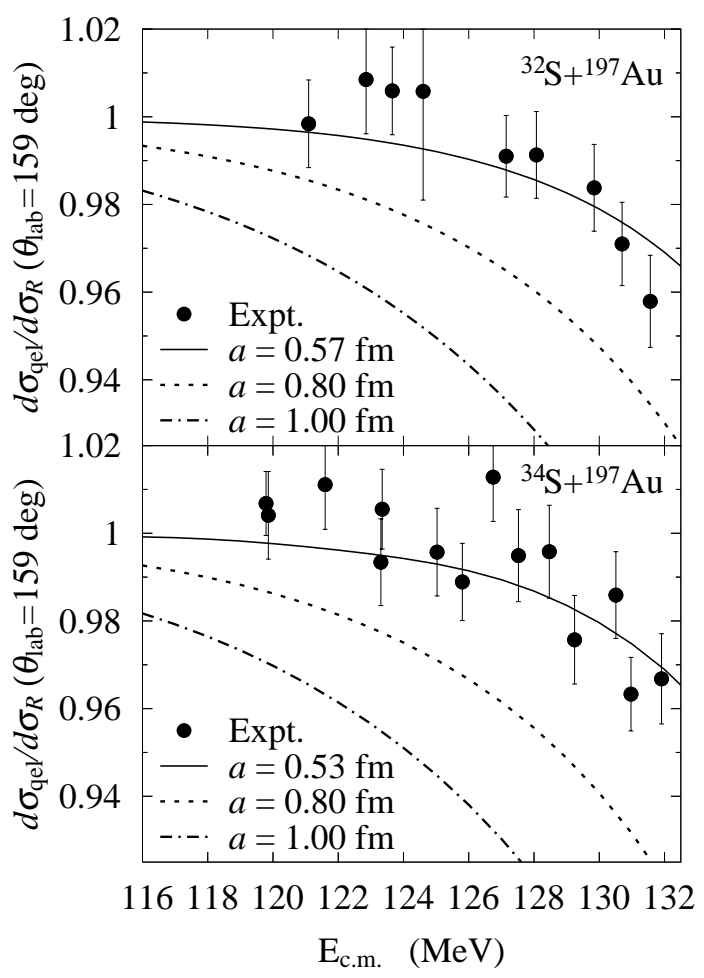

FIGURE 1. The ratio of the quasi-elastic to the Rutherford cross sections at $\theta_{\mathrm{lab}}=159^{\circ}$ for the ${ }^{32} \mathrm{~S}+$ ${ }^{197} \mathrm{Au}$ (the upper panel) reaction and for the ${ }^{34} \mathrm{~S}+{ }^{197} \mathrm{Au}$ (the lower panel) reaction.

elastic cross sections from the Rutherford ones is sensitive predominantly to the surface region of the nuclear potential, especially to the surface diffuseness parameter $a$. There is another advantage of using the deep sub-barrier data. That is, the effect of channel coupling on quasi-elastic scattering can be disregarded at these energies, since the reflection probability is almost unity irrespective of the presence of channel couplings [14]. From these considerations, it is evident that the effect of surface diffuseness parameter can be studied in a transparent and unambiguous way using the large-angle quasi-elastic scattering at deep sub-barrier energies.

Figure 1 compares the experimental data with the calculated cross sections obtained with different values of the surface diffuseness parameter for the ${ }^{32} \mathrm{~S}+{ }^{197} \mathrm{Au}$ system (the upper panel) and the ${ }^{34} \mathrm{~S}+{ }^{197} \mathrm{Au}$ system (the lower panel). In order to analyze the experimental data at deep sub-barrier energies, we use a one-dimensional optical potential with the Woods-Saxon form. Absorption following transmission through the barrier is simulated by an imaginary potential that is well localized inside the Coulomb barrier. The best fitted values for the surface diffuseness parameter are $a=0.57 \pm 0.04$ fm and $a=0.53 \pm 0.03 \mathrm{fm}$ for the ${ }^{32} \mathrm{~S}$ and ${ }^{34} \mathrm{~S}+{ }^{197} \mathrm{Au}$ reactions, respectively. The cross sections obtained with these surface diffuseness parameters are denoted by the solid line in the figure. The dotted and the dot-dashed lines are calculated with the diffuseness parameter of $a=0.80 \mathrm{fm}$ and $a=1.00 \mathrm{fm}$, respectively. It is clear from the figure that these spherical systems favor the standard value of the surface diffuseness parameter, around $a=0.60 \mathrm{fm}$. The calculations with the larger diffuseness parameters 
underestimate the quasi-elastic cross sections and are not consistent with the energy dependence of the experimental data. We obtain a similar conclusion for the ${ }^{32,34} \mathrm{~S}+$ ${ }^{208} \mathrm{~Pb}$ and ${ }^{16} \mathrm{O}+{ }^{208} \mathrm{~Pb}$ systems[13]. This indicates that the double folding procedure is valid at least in the surface region and for spherical systems which we studied.

For deformed systems, such as ${ }^{16} \mathrm{O}+{ }^{154} \mathrm{Sm},{ }^{186} \mathrm{~W}$, on the other hand, we found that the surface diffuseness parameter of $a=1.14 \pm 0.03 \mathrm{fm}$ and $0.79 \pm 0.04 \mathrm{fm}$ for the former and for the latter, respectively, is required in order to account for the experimental data [13, 14]. Although these large values of surface diffuseness parameter are consistent with that extracted from fusion, the origin of the difference between the spherical and the deformed systems is not clear. In order to clarify the difference in the diffuseness parameter, apparently further precision measurements for large-angle quasi-elastic scattering at deep sub-barrier energies are urged, especially for deformed systems.

\section{COUPLED-CHANNELS CALCULATIONS FOR QUASI-ELASTIC BARRIER DISTRIBUTION FOR ${ }^{70} \mathrm{ZN}+{ }^{208}$ PB REACTION}

We next discuss the barrier distribution for synthesis of superheavy elements. When one discusses a fusion reaction to synthesize superheavy elements, one often refers to a single potential such as the Bass barrier [22]. On the other hand, the effect of channel coupling is in general strong for massive systems, and thus one can expect a broad distribution of potential barriers. It is thus important to study how the potential barrier is distributed for massive systems, since it is crucial to choose the right beam energy in order to effectively synthesize superheavy elements. Moreover, there is no a priori evidence why the Bass barrier is reasonable in the superheavy region. For these reasons, the quasi-elastic barrier distribution measurements have been recently performed by Mitsuoka et al. for systems relevant to cold fusion reactions, ${ }^{48} \mathrm{Ti},{ }^{54} \mathrm{Cr},{ }^{56} \mathrm{Fe},{ }^{64} \mathrm{Ni},{ }^{70} \mathrm{Zn}$ $+{ }^{208} \mathrm{~Pb}$ [15]. In this section, we perform coupled-channels calculations for the ${ }^{70} \mathrm{Zn}+$ ${ }^{208} \mathrm{~Pb}$ system.

The calculations are done with a version [23] of the coupled-channels code CCFULL [24]. This code treats the coupling to all orders in the coupling hamiltonian and employs the isocentrifugal approximation in order to reduce the dimension of the coupledchannels equations. It has been shown that the isocentrifugal approximation works well for quasi-elastic scattering at backward angles [12]. In the code, the regular boundary condition is imposed at the origin, instead of the incoming boundary condition.

Figure 2 shows the excitation function of the quasi-elastic scattering (the upper panel) and the barrier distribution (the lower panel). The deep-inelastic component has been subtracted from the experimental data using a statistical code, as is explained in Ref. [15]. The solid and dashed lines are the results of the coupled-channels and the potential model calculations, respectively. We use the Woods-Saxon potential with $V_{0}=140 \mathrm{MeV}$, $r_{0}=1.186 \mathrm{fm}$, and $a=0.69 \mathrm{fm}$ for the real part and $W_{0}=50.0 \mathrm{MeV}, r_{w}=1.0 \mathrm{fm}$, and $a_{w}=$ $0.1 \mathrm{fm}$ for the imaginary part. In the coupled-channels calculation, we include the double quadrupole phonon excitations in the ${ }^{70} \mathrm{Zn}$ and the triple octupole phonon excitations in the ${ }^{208} \mathrm{~Pb}$ nucleus. In addition, we include the mutual excitation channels, [1,1], [1,2], $[2,1]$, and $[2,2]$, where $\left[n_{P}, n_{T}\right]$ denotes the excitation channel with $n_{P}$ phonon state in the projectile and $n_{T}$ phonon state in the target nucleus. In this way, we include 10 channels 


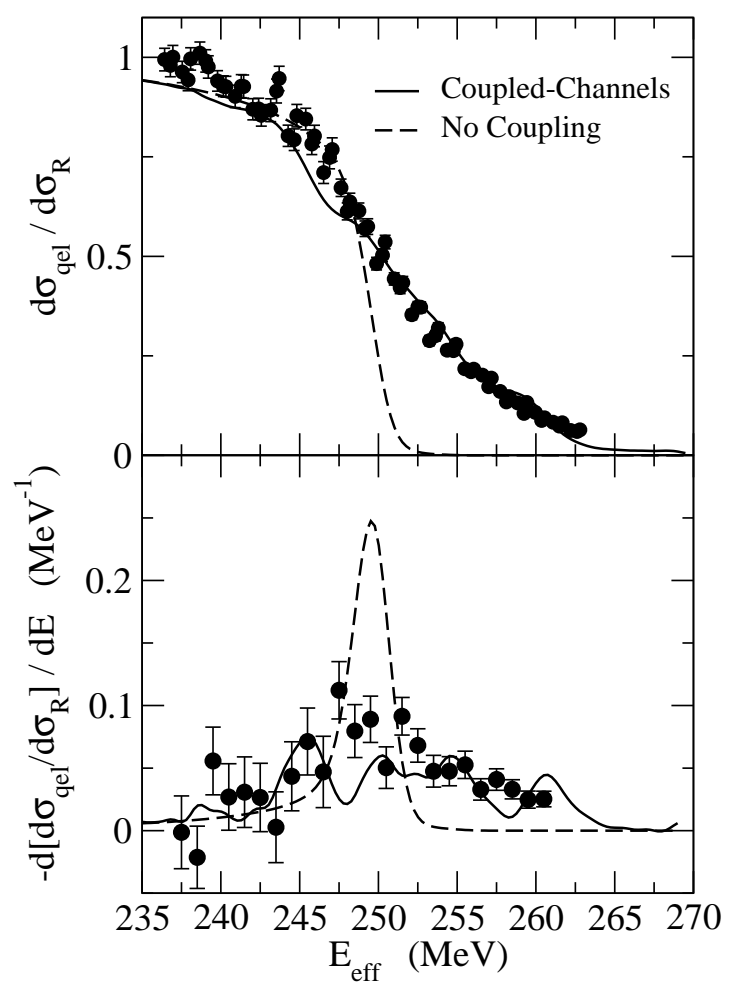

FIGURE 2. The ratio of the quasi-elastic to the Rutherford cross sections (the upper panel) and the quasi-elastic barrier distribution (the lower panel) for the ${ }^{70} \mathrm{Zn}+{ }^{208} \mathrm{~Pb}$ reaction. These are plotted as a function of effective energy defined by Eq. (8). The solid line is the solution of coupled-channels equations, which take into account the double quadrupole phonon excitations in the ${ }^{70} \mathrm{Zn}$ nucleus and the triple octupole phonon excitations in the ${ }^{208} \mathrm{~Pb}$ nucleus. The dashed line shows the result without the couplings. The experimental data are taken from Ref. [15].

(including the entrance channel, $[0,0])$ in the calculations. The excitation energy for the single phonon state and the deformation parameter are $E_{2^{+}}=0.885 \mathrm{MeV}$ and $\beta_{2}=0.228$ for the projectile nucleus ${ }^{70} \mathrm{Zn}$ and $E_{3^{-}}=2.614 \mathrm{MeV}$ and $\beta_{3}=0.11$ for the target nucleus ${ }^{208} \mathrm{~Pb}$. We use $r_{0}=1.2 \mathrm{fm}$ for the coupling term.

In the code, the coupled-channels equations are solved by constructing $N$ linear independent solutions of the equations, where $N$ is the dimension of the coupled-channels equations. A linear superposition of these solutions is then taken to construct the physical solution, which fulfills the asymptotic boundary condition for scattering. For massive systems, it is sometimes difficult to numerically maintain the linear independence of the solutions, since the wave functions scale very differently from one channel to another. This leads to a numerical instability of the solution of the coupled-channels equations. We avoid this difficulty by taking a linear superposition of the solutions at several places, with an interval of $1 \mathrm{fm}$ up to $15 \mathrm{fm}$, so that the linear independence is recovered. See Ref. [23] for details. Even though we use this prescription, we still find a small spurious oscillation in the calculated excitation function of quasi-elastic cross section due to the numerical inaccuracy, when the coupling is strong. We therefore average the calculated cross sections with a Gaussian weight with $0.5 \mathrm{MeV}$ width. We have checked 


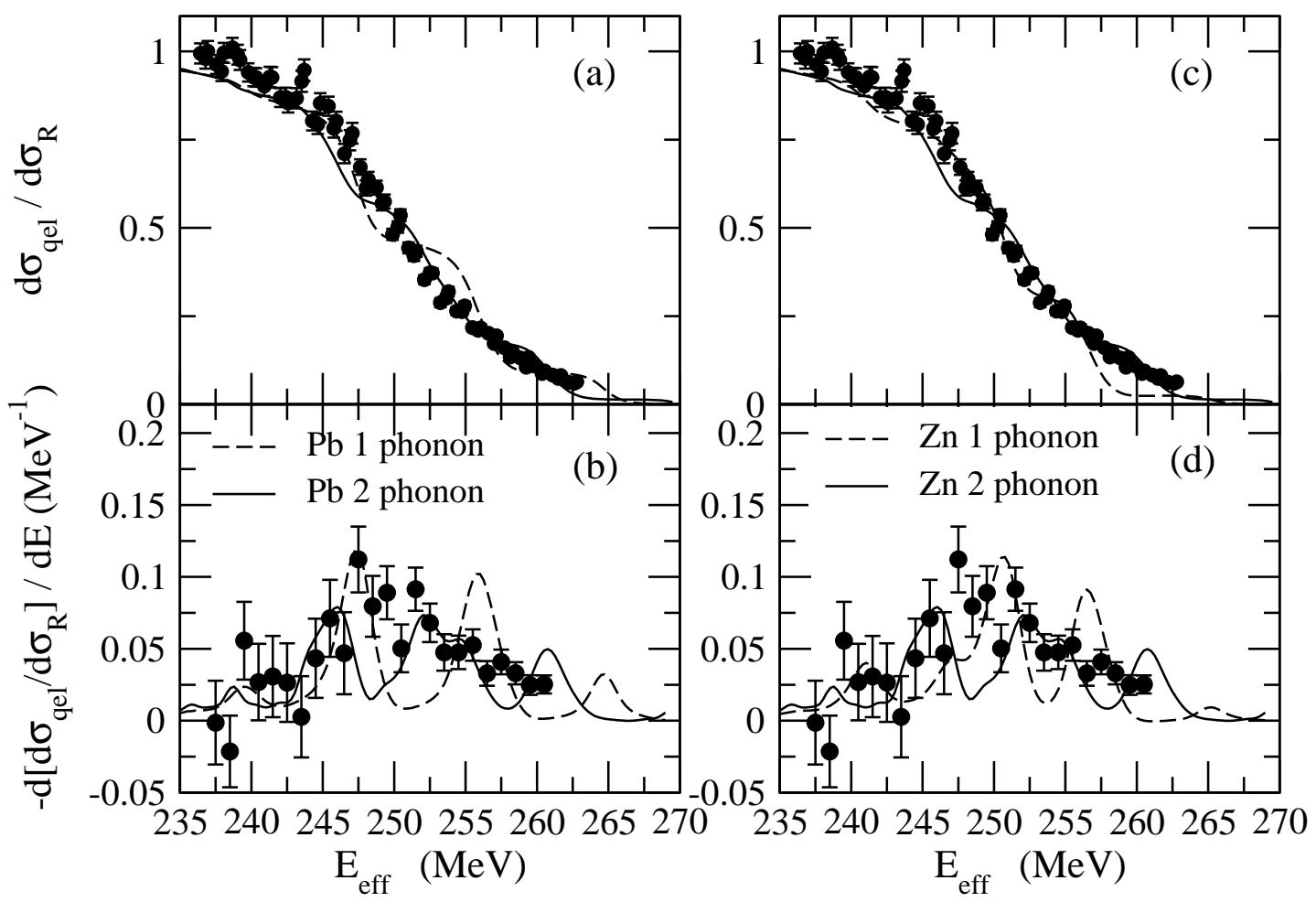

FIGURE 3. The ratio of the quasi-elastic to the Rutherford cross sections (3(a) and 3(c)) and the quasielastic barrier distribution (3(b) and 3(d)) for the ${ }^{70} \mathrm{Zn}+{ }^{208} \mathrm{~Pb}$ reaction. The figs. 3(a) and 3(b) are obtained by including the different number of octupole phonon excitations in the target nucleus as indicated in the inset, together with the double quadrupole phonon excitations in the projectile nucleus. The figs. 3(c) and 3 (d) are obtained by including the different number of quadrupole phonon excitations in the projectile nucleus together with the double octupole phonon excitations in the target nucleus.

that the shape of quasi-elastic barrier distribution is insensitive to the value of the width parameter.

As we can see in the figure, the potential model calculation (the dashed line) significantly underestimate the quasi-elastic cross sections at energies above the Coulomb barrier. Also, the barrier distribution has a significantly narrow width, and is inconsistent with the experimental data. On the other hand, the coupled-channels calculation (the solid line) well reproduces the experimental data both for the excitation function and barrier distribution.

Figure 3 shows the role of multi-phonon excitations. Figures 3(a) and 3(b) are obtained by varying the number of octupole phonon excitations in the target while keeping the double phonon excitations in the projectile nucleus. On the other hand, figs. 3(c) and 3(d) are obtained by varying the number of quadrupole phonon excitation while keeping the number of octupole phonon excitations in the target nucleus to be two. These figures show that the double phonon excitations considerably alter the shape of barrier distribution as compared with the barrier distribution obtained with the single phonon excitation. For both in the projectile and in the target nuclei, the double phonon excitation leads to better agreement with the experimental data, although we find that the triple phonon ex- 
citation in the target nucleus plays a much less important role. A similar conclusion has been obtained also in Ref.[10], where the role of multi-phonon excitations was discussed for the ${ }^{100} \mathrm{Mo}+{ }^{100} \mathrm{Mo}$ fusion reaction at energies around the Coulomb barrier.

\section{SUMMARY}

We have discussed two recent developments in heavy-ion quasi-elastic scattering at energies around the Coulomb barrier. We first discussed the surface property of internucleus potential. We have argued that the quasi-elastic scattering at deep subbarrier energies offer a clear and almost model independent way to determine the surface diffuseness parameter, that is, the slope of asymptotic exponential tail of the potential. The value of diffuseness parameter extracted from the ${ }^{32,34} \mathrm{~S}+{ }^{197} \mathrm{Au}$ reactions is around $0.55 \mathrm{fm}$, and is consistent with the double folding potential. On the other hand, the surface diffuseness parameter extracted from systems with a deformed target, that is, ${ }^{16} \mathrm{O}+{ }^{154} \mathrm{Sm},{ }^{186} \mathrm{~W}$ was found to be much larger (1.14 fm for the former and $0.79 \mathrm{fm}$ for the latter systems). Further investigations will be required in order to clarify the system dependence of the surface diffuseness parameter. In the second part, we performed the coupled-channels analyses for a cold fusion reaction ${ }^{70} \mathrm{Zn}+{ }^{208} \mathrm{~Pb}$, where the quasi-elastic barrier distribution was recently obtained by Mitsuoka et al.. Including the double quadrupole phonon excitations in the projectile nucleus ${ }^{70} \mathrm{Zn}$ and the triple octupole phonon excitations in the target nucleus ${ }^{208} \mathrm{~Pb}$ in the coupled-channels calculation, we could reproduce reasonably well both the excitation function of quasi-elastic cross section and the shape of quasi-elastic barrier distribution. This indicates that the coupled-channels approach still works for the approaching phase of the reaction even in massive systems, where many degrees of freedom may be involved in the reaction [10]. It also suggests that the deepinelastic collision can be regarded as a post-barrier phenomena, since the experimental quasi-elastic cross sections have been obtained by subtracting the deep-inelastic components. We also discussed the role of multi-phonon excitations, and showed that they play an important role in this system. The coupled-channels analyses for other cold fusion reactions, ${ }^{48} \mathrm{Ti},{ }^{54} \mathrm{Cr},{ }^{56} \mathrm{Fe},{ }^{64} \mathrm{Ni}+{ }^{208} \mathrm{~Pb}$ are now in progress, and we will report on them in a separate publication.

\section{ACKNOWLEDGMENTS}

This work is based on collaborations with N. Rowley and K. Washiyama. We thank H. Ikezoe and S. Mitsuoka for useful discussions and for sending us the experimental data before publication. This work was supported by the Grant-in-Aid for Scientific Research, Contract No. 16740139 from the Japanese Ministry of Education, Culture, Sports, Science, and Technology.

\section{REFERENCES}

1. C.Y. Wong, Phys. Rev. Lett. 31, 766 (1973).

2. M.V. Andres, N. Rowley, and M.A. Nagarajan, Phys. Lett. 202B, 292 (1988). 
3. T. Rumin, K. Hagino, and N. Takigawa, Phys. Rev. C63, 044603 (2001).

4. M. Dasgupta, D.J. Hinde, N. Rowley, and A.M. Stefanini, Annu. Rev. Nucl. Part. Sci. 48, 401 (1998).

5. A.B. Balantekin and N. Takigawa, Rev. Mod. Phys. 70, 77 (1998).

6. K. Hagino and A.B. Balantekin, Phys. Rev. A70, 032106 (2004).

7. C.H. Dasso, S. Landowne, and A. Winther, Nucl. Phys. A405, 381 (1983); A407, 221 (1983).

8. N. Rowley, G.R. Satchler, and P.H. Stelson, Phys. Lett. B254, 25 (1991).

9. J.R. Leigh et al., Phys. Rev. C52, 3151 (1995).

10. N. Rowley, N. Grar, and K. Hagino, Phys. Lett. B632, 243 (2006).

11. H. Timmers et al., Nucl. Phys. A584, 190 (1995).

12. K. Hagino and N. Rowley, Phys. Rev. C69, 054610 (2004); Brazilian J. Phys. 35, 890 (2005).

13. K. Washiyama, K. Hagino, and M. Dasgupta, Phys. Rev. C73, 034607 (2006).

14. K. Hagino, T. Takehi, A. B. Balantekin, and N. Takigawa, Phys. Rev. C 71, 044612 (2005).

15. H. Ikezoe, S. Mitsuoka, K. Nishio, K. Tsuruta, Y. Watanabe, S. Jeong, and K. Sato, in proceedings of international conference "FUSION06", AIP conf. proc. 853, 69 (2006); S. Mitsuoka, private communications.

16. K. Hagino et al., in proc. of the 4th Italy-Japan symposium on Heavy-Ion Physics, edited by S. Kubono et al. (World Scientific, Singapore, 2002), p.87. e-print: nucl-th/0110065.

17. R.A. Broglia and A. Winther, Heavy Ion Reactions, Vol. 84 in Frontiers in Physics Lecture Note Series (Addison-Wesley, Redwood City, CA, 1991).

18. P.R. Christensen and A. Winther, Phys. Lett. 65B, 19 (1976).

19. G.R. Satchler and W.G. Love, Phys. Rep. 55, 183 (1979).

20. J.O. Newton et al., Phys. Lett. B586, 219 (2004); Phys. Rev. C 70, 024605 (2004).

21. S. Landowne and H.H. Wolter, Nucl. Phys. A351, 171 (1981).

22. R. Bass, Phys. Rev. Lett. 39, 265 (1977); Nuclear Reactions with Heavy Ions, (Springer Verlag, Berlin Heidelberg New York, 1980) p. 152.

23. K. Hagino and N. Rowley, to be published.

24. K. Hagino, N. Rowley, and A.T. Kruppa, Comp. Phys. Comm. 123, 143 (1999). 